NASA Contractor Report 182083

ICASE Report No. 90-55

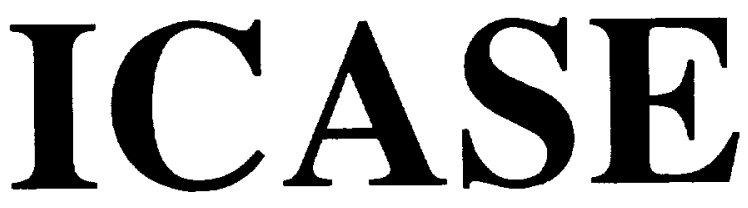

NUMERICAL EXPERIMENTS ON THE ACCURACY
OF ENO AND MODIFIED ENO SCHEMES

Chi-Wang Shu

Contract No. NAS1-18605

August 1990

Institute for Computer Applications in Science and Engineering NASA Langley Research Center

Hampton, Virginia 23665-5225

Operated by the Universities Space Research Association

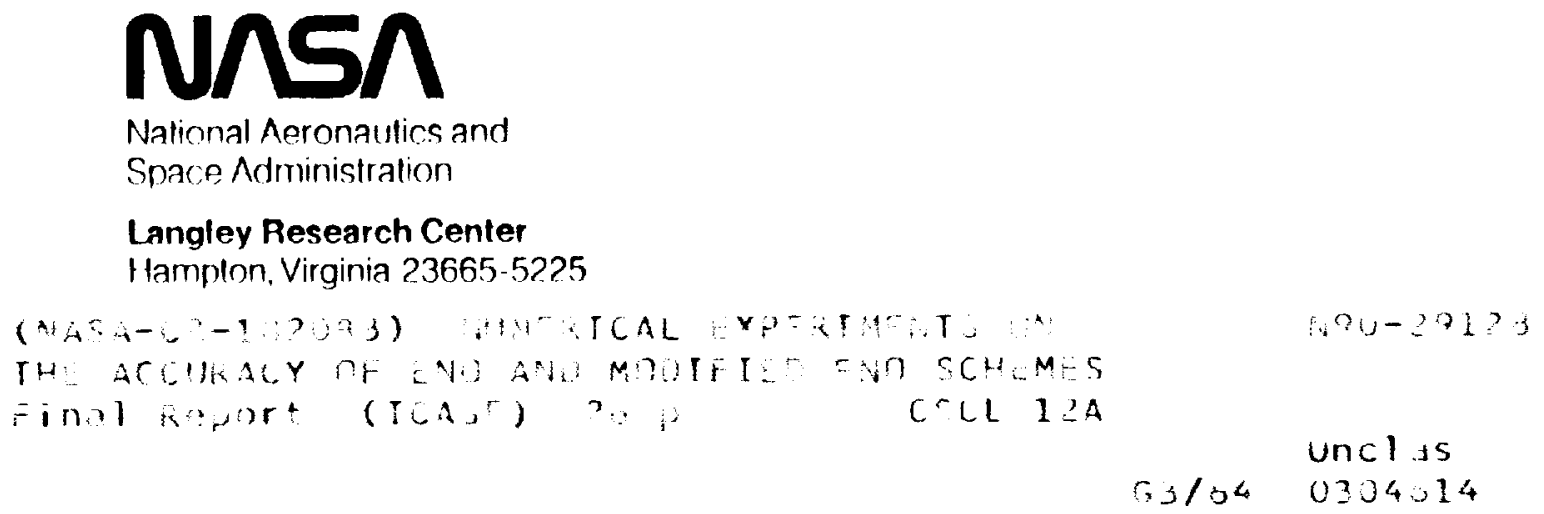




\title{
NUMERICAL EXPERIMENTS ON THE ACCURACY OF ENO AND MODIFIED ENO SCHEMES
}

\author{
Chi-Wang Shu ${ }^{1}$ \\ Division of Applied Mathematics \\ Brown University \\ Providence, RI 02912
}

\begin{abstract}
In this paper we make further numerical experiments assessing an accuracy degeneracy phenomena reported by A. Rogerson and E. Meiburg [7]. We also propose a modified ENO scheme, which recovers the correct order of accuracy for all the test problems with smooth initial conditions and gives comparable results with the original ENO schemes for discontinuous problems.
\end{abstract}

\footnotetext{
${ }^{1}$ Research was supported by the National Aeronautics and Space Administration under NASA Contract No. NAS1-18605 while the author was in residence at the Institute for Computer Applications in Science and Engineering (ICASE), NASA Langley Research Center, Hampton, VA 23665. Research also supported by NSF Grant DMS88-10150 and AFOSR Grant 90-0093.
} 



\section{Introduction}

ENO (essentially non-oscillatory) schemes were first developed by Harten and Osher [2], Harten, Engquist, Osher and Chakravarthy [3], [4], to solve a hyperbolic conservation law

$$
\left\{\begin{array}{l}
u_{t}+f(u)_{x}=0 \\
u(x, 0)=u^{0}(x)
\end{array}\right.
$$

whose solution may be discontinuous even if the initial condition $u^{0}(x)$ is smooth. The philosophy of ENO schemes is to use an adaptive stencil, based on local smoothness, to automatically avoid interpolations across discontinuities. As a result, a formally uniform high order of accuracy, measured by local truncation errors, and sharp, essentially nonoscillatory shock transitions can be obtained. Shu and Osher [8], [9] later proposed efficient ways of implementing ENO schemes based on numerical fluxes and a special class of TVD (total-variation-diminishing) Runge-Kutta type high order time discretizations, which uses a conservative flux difference

$$
\frac{1}{\Delta x}\left(\hat{f}_{j+\frac{1}{2}}-\hat{f}_{j-\frac{1}{2}}\right)
$$

to approximate $f(u)_{x}$ in (1.1) to high order, where the numerical fluxes $\hat{f}$ are evaluated using high order interpolation polynomials constructed from adaptive stencils.

Even if the numerical examples in [3],[8],[9] are very impressive, a convergence theory for ENO schemes is still not available, due to the nonlinearity of the scheme. A more difficult problem is to prove any convergence rate in smooth regions, since total variation stability, a tool used very often for proving convergence of nonlinear schemes in one space dimension, does not provide such a rate of convergence.

Recently, A. Rogerson and E. Meiburg reported in [7] that for a linear, constant-coefficient version of (1.1):

$$
\left\{\begin{array}{l}
u_{t}+u_{x}=0 \\
u(x, 0)=u^{0}(x)
\end{array}\right.
$$

the convergence rate is dependent upon the initial condition $u^{0}(x)$ (assumed smooth) and may be inferior to what is predicted by the local truncation error analysis. Furthermore the error reduction during mesh refinements is not uniform and there are cases where a refined mesh gives a larger error. The source of this phenomena is traced to the fact that a linearly unstable stencil is initially chosen by ENO in a large portion of the domain. It was known to Harten et al [3] that the use of linearly unstable stencils will lead to frequent stencil switchings and loss of one order of accuracy due to the failure of error cancellation in the conservative form (1.2) (they verified this by using the initial condition $u^{0}(x)=e^{-x}$ in (1.3) 
so that the initial stencil chosen by ENO is always the linearly unstable downwind stencil). The results in [7] indicates that the accuracy degeneracy phenomena can be more serious.

In this paper, we perform further numerical experiments, including the use of different ENO schemes (cell-averaged version [3],[4] and the flux-pointvalue verion [8],[9]) and different time discretizations (TVD Runge-Kutta [8],[9] and exact time evolution [4]), to assess the accuracy degeneracy phenomena more thoroughly. We also propose a modified ENO scheme, which (1) introduces no additional computational cost over the original ENO scheme; (2) recovers the correct order of accuracy for (1.3) with different smooth initial conditions; and (3) gives comparable results with the original ENO schemes for discontinuous problems.

All the computations in this paper are performed on a Cray- 2 supercomputer. The largest number of grid points used is 5120 .

\section{Numerical Experiments with ENO Schemes}

We use the third order ENO scheme based on numerical fluxes, and the third order TVD Runge-Kutta time discretization, described in detail in [8],[9]. CFL number is chosen as $\frac{\Delta t}{\Delta x}=0.6$ and the terminal time in (1.3) is chosen as $t=4$ (two time periods). As in [7], We choose the initial conditions $u^{0}(x)$ in $(1.3)$ as periodic functions with period 2 , in order to exclude possible degeneracy of accuracy due to boundary conditions.

First we briefly revisit the two examples used in [7], i.e. $u^{0}(x)=\sin (\pi x)$ and $u^{0}(x)=$ $\sin ^{4}(\pi x)$, using the more commonly used third order in space and time ENO scheme with $C F L=0.6$. As in [7], we can see that ENO is as accurate as the linearly stable centered scheme for $u^{0}(x)=\sin (\pi x)$ (Figure 1a), but for $u^{0}(x)=\sin ^{4}(\pi x)$ the $L_{1}$ error of the ENO scheme no longer monotonically decreases with the mesh size, and the final asymptotic order of accuracy is around 1.97, which is less than 3, predicted by local truncation error analysis (Figure 2a). By monitoring the stencil as in [7], we can see that, for $u^{0}(x)=\sin (\pi x)$, the initial stencil chosen by ENO falls completely inside the linear stability range 0 and -1 (Figure 1b), and it changes very little after two time periods (Figure 1c). On the other hand, for $u^{0}(x)=\sin ^{4}(\pi x)$, ENO initially picks the linearly unstable stencils 1 and -2 in a large portion of the domain (Figure $2 b$ ) and then switches its stencil violently in order to balance the linear instability effects (Figures 2c and 2d). To see the different behavior of ENO scheme and the stable and unstable linear schemes, in Figure $2 \mathrm{e}$ we plot the logorithm of the $L_{1}$ error versus time for 160 grid points (which is the worst case for ENO from Figure 2a). We can see that for a short time the $L_{1}$ error of ENO grows exponentially, similar to that of the linearly unstable scheme, but later ENO quickly stablizes itself, apparently through the nonlinear effect of stencil switching, while the linearly unstable scheme simply 
blows up. However, what is lost to the accuracy during the brief exponential growth is not recovered, resulting in a $L_{1}$ error for ENO which for this case is almost two magnitudes larger than that of the linearly stable centered scheme.

Our next example is $u^{0}(x)=e^{-x}$. This is a discontinuous initial condition since we enforce periodicity. We compute the $L_{1}$ error in the smooth region $-0.5 \leq x \leq 0.5$ at $t=4$. From Figures $3 \mathrm{a}$ and $3 \mathrm{e}$ we can see that the linearly stable centered scheme now performs very poorly, with large Gibbs oscillations near the discontinuity and only first order accuracy even in the smooth region, due to the pollution of Gibbs oscillations. For this particular example the pollution can be avoided by using an exact time evolution, but for general nonlinear problems this pollution is very typical to high order linearly stable schemes applied to discontinuous problems. ENO scheme behaves much better than the linear scheme, cf Figures 3a and 3f, however we still observe the non-uniform decaying of errors with the mesh refinements, similar to the previous case $u^{0}(x)=\sin ^{4}(\pi x)$. This example was used in [3], without periodicity assumption, to illustrate the self-correcting mechanism of ENO schemes. Exact time evolution was used in [3], which explains the difference there and in Figure 3a. ¿From Figure 3b we can see, as expected, that the initial stencil chosen by ENO is almost always the linearly unstable 1 , and from Figures $3 \mathrm{c}$ and $3 \mathrm{~d}$ we again observe the violent stencil switching at later times.

Other initial conditions, such as $u^{0}(x)=\sin ^{10}(\pi x), u^{0}(x)=\left(x^{2}-1\right)^{4}$ enforcing periodicity, $u^{0}(x)=e^{\sin (\pi x)}, u^{0}(x)=\sin (\cos (\pi x))$, etc., have been tested as well. We observe this degeneracy phenomena, to different extent, for all those cases.

One naturally suspects whether this phenomena is related to the Runge-Kutta time stepping. As a matter of fact the result for ENO does become better with an exact time evolution [3], especially for $u^{0}(x)=e^{-x}$. However, for most of the cases we have tested, this difference is only quantitative: the accuracy degeneracy phemonena is still present. Figure 4a shows the result with exact time evolution for $u^{0}(x)=\sin ^{4}(\pi x)$. Comparing with Figure 2a, we can see that the error of ENO now decays monotonically with mesh refinements, and the final asymptotic order of convergence is around 2.5 , which is better than the Runge-Kutta case but still shows the degeneracy of accuracy. This suggests that the root of this degeneracy is the ENO spactial operator, although Runge-Kutta time stepping may compound the problem.

We have also tested the original cell-averaged ENO schemes in [3], with the same initial conditions. We have not observed essential differences. Compare, e.g., Figure $4 \mathrm{~b}$ with Figure 4a. For the equation (1.3), the only difference between the two versions of ENO schemes is the initial condition (cell-averages versus point values).

Different spatial orders for ENO schemes are also tested: up to seventh order with exact 
time evolution. The accuracy degeneracy phenomena is present for all orders, but is getting less serious when the order increases. Compare, e.g., Figure 4c with Figure 4a. At seventh order (Figure 4c) the ENO result is quite satisfactory in the practical range of the number of grid points. This suggests that for a fixed cost, a higher order ENO with fewer grid points may be preferred to a lower order one with more points.

Finally, the effect of this phenomena with different terminal time is tested. Figure $4 \mathrm{~d}$ $(t=8)$ should be compared with Figure $2 \mathrm{a}(t=4)$. We clearly see qualitatively similar results.

\section{A Modification of ENO Schemes}

The philosophy of ENO schemes is to adaptively choose stencils so that interpolations across discontinuities can be avoided. In smooth regions there seems to be conceptionally no need to choose linearly unstable stencils in the stencil-choosing procedure. The numerical evidence in [7] and in the previous section suggests that it is even harmful to the accuracy. Consequently some strategy to use a simpler fixed stencil linearly stable scheme in the smooth regions and to use ENO near discontinuities seems to be appealing. Earlier work for such strategies has already been documented in e.g., [5] and [10], with a motivation to save computational costs. These approaches will also solve the accuracy degeneracy problems for the examples with smooth initial conditions in [7] and in the previous section, since a linearly stable centered scheme is used throughout the region except for possibly a few isolated cells near critical points, which seems not to affect the accuracy in the numerical tests (S. Osher, private communication).

In this section we discuss a modification of ENO schemes which involves only a slight change in the coding, without increasing the computational costs. A similar modification

has been used in [1] for a different purpose (getting smoother steady state solutions). Instead of using

$$
\left\{\begin{array}{l}
i(j)=j \quad \text { (upwinding }) \\
\text { for } k=2, \ldots, r+1: \\
\text { if }(a b s(H[i(j), k]) \cdot g t . a b s(H[i(j)-1, k])) i(j)=i(j)-1 \\
\text { end for }
\end{array}\right.
$$

to determine the left-most point $i(j)$ in the stencil for computing the numerical flux $\hat{f}_{j+\frac{1}{2}}$ in (1.2) $(H[i, k]$ is the $\mathrm{k}$-th divided (or undivided during the actual coding) difference of the 
function $H(x)$, whose definition and relations to $f(u(x))$ can be found in [9]), we use instead

$$
\left\{\begin{array}{l}
i(j)=j \quad \text { (upwinding) } \\
\text { for } k=2, \ldots, r+1: \\
\text { if }\left(i(j) \cdot g t \cdot i_{c}(j)\right) \text { then } \\
\text { if }(2 * a b s(H[i(j), k]) \cdot g t . a b s(H[i(j)-1, k])) i(j)=i(j)-1 \\
\text { else } \\
\text { if }(a b s(H[i(j), k]) \cdot g t .2 * a b s(H[i(j)-1, k])) i(j)=i(j)-1 \\
\text { end if } \\
\text { end for }
\end{array}\right.
$$

where $i_{c}(j)$ is the left-most point in the linearly stable centered stencil. By using this modification, we are trying to bias towards the linearly stable centered stencil as much as possible in the smooth regions. Since the ratio of the two differences being compared in (3.1) is $1+O(\Delta x)$ in smooth regions, it is easy to prove that in smooth regions where all the derivatives of $H(x)$ are non-zeroes, (3.2) will give the centered stencil when $\Delta x$ is sufficiently small. Near shocks the modifications should not affect the non-oscillatory philosophy, since the differences being compared are then of different magnitudes.

We first test the performance of the modified ENO scheme (3.2) on the examples in [7] and in the previous section. As one can see from Figures $1 \mathrm{a}$ and $2 a$, the modified ENO is fully third order accurate and is in fact indistinguishable from the linearly stable centered scheme for smooth initial conditions. From Figures $3 \mathrm{a}$ and $3 \mathrm{~g}$ we can see that the modified ENO performs much better than the linearly stable centered scheme and better than the ENO scheme, by producing fully third order accuracy in smooth regions and a non-oscillatory shock transition, when the initial condition is discontinuous. From Figures $2 \mathrm{f}, 2 \mathrm{~g}$ and $3 \mathrm{~h}, 3 \mathrm{i}$ we can see that, in the smooth regions, the stencils of the modified ENO scheme are almost always within the linearly stable choices 0 and -1 . This is probably the main reason for its excellent behavior. We have also tested the modified ENO scheme in many other cases, such as all those mentioned in the previous section. Full high order of accuracy predicted by local truncation error analysis is always observed.

We remark here that (3.2) with $r=1$ is a MUSCL type second order (in the $L_{1}$ sense) TVD scheme discussed in [6] (with the minmod function replaced by a minimum-in-absolutevalue function). This is a major reason for our choice of the factor 2 . Any factor bigger than 1 should have the same effect of biasing towards a centered stencil asymptotically. We do not recommend a factor bigger than 2 since it will not produce a TVD scheme for $r=1$.

A more important test for the performance of the modified ENO scheme is to apply it to problems with both discontinuities and detailed smooth structures. we have already seen the application to (1.3) with $u^{0}(x)=e^{-x}$ in Figures $3 \mathrm{a}$ and $3 \mathrm{~g}$. We now apply it to the one dimensional Euler equations of gas dynamics, which is a nonlinear hyperbolic 
system. We choose two shock tube problems and a problem of shock interaction with density perturbations, see [9] for details of equations and parameters. Comparisons between Figures $3 \mathrm{f}$ and $3 \mathrm{~g}, 5 \mathrm{a}$ and $5 \mathrm{~b}$, and $6 \mathrm{a}$ and $6 \mathrm{~b}$, reveal almost identical performance of ENO and modified ENO schemes. For the more interesting test case of shock interaction with density perturbations, Figures $7 \mathrm{a}$ and $7 \mathrm{~b}$, we observe a slightly better resolution of the modified ENO versus ENO with 160 grid points, which is marginal for ENO to resolve this structure. However, the modified ENO has a slight over-compression effect (step phenomena) in the smooth regions, which is more visible in Figure $7 \mathrm{~d}$ with 400 grid points. This is anticipated since (3.2), in many cases (e.g. for $r=1$ ), is similar to the artificial compression used in [10] and [9].

\section{Concluding Remarks}

ENO schemes may lose the full high order accuracy predicted by local truncation error analysis, for scalar linear conservation laws with smooth initial conditions. A modified ENO scheme, which does not increase the computational cost, can overcome this accuracy degeneracy problem for the test problems. For systems of nonlinear conservation laws with solutions containing both shocks and detailed smooth structures, ENO and modified ENO perform comparably, with the modified ENO having a slightly better resolution but showing some over-compression effects.

Acknowledgment: We thank Stanley Osher for many valuable suggestions. We also thank David Gottlieb, Eckart Meiburg and Andrey Rogerson for helpful discussions. 


\section{References}

[1] E. Fatemi, J. Jerome and S. Osher, Solution of the hydrodynamic device model using high order non-oscillatory shock capturing algorithms, ICASE Report 89-52, NASA Langley Research Center, 1989. To appear in IEEE Transactions on Computer Audeo Design of Integrated Systems and Circuits.

[2] A. Harten and S. Osher, Uniformly high order accurate nonoscillatory schemes, I, SIAM J. Numer. Anal., v.24, 1987, pp.279-309.

[3] A. Harten, B. Engquist, S. Osher and S. Chakravarthy, Uniformly high order essentially non-oscillatory schemes, III, J. Comput. Phys., v.71, 1987, pp.231-303.

[4] A. Harten, S. Osher, B. Engquist and S. Chakravarthy, Some results on uniformly high order accurate essentially non-oscillatory schemes, J. Appl. Numer. Math., v.2, 1986, pp.347-377.

[5] F. Lafon and S. Osher, High order filtering methods for approximating heperbolic systems of conservation laws, ICASE Report 90-25, NASA Langley research Center, 1990. Submitted to J. Comput. Phys.

[6] S. Osher, Convergence of generalized MUSCL schemes, SIAM J. Numer. Anal., v.22, 1985, pp.947-961.

[7] A. Rogerson and E. Meiburg, A numerical study of the convergence properties of ENO schemes, preprint. Submitted to J. Sci. Comput.

[8] C.-W. Shu and S. Osher, efficient implementation of essentially non-oscillatory shock capturing schemes, J. Comput. Phys., v.77, 1988, pp.439-471.

[9] C.-W. Shu and S. Osher, efficient implementation of essentially non-oscillatory shock capturing schemes, II, J. Comput. Phys., v.83, 1989, pp.32-78.

[10] H. Yang, Some results on numerical methods for hyperbolic conservation laws, Ph.D Thesis, UCLA, 1989. 


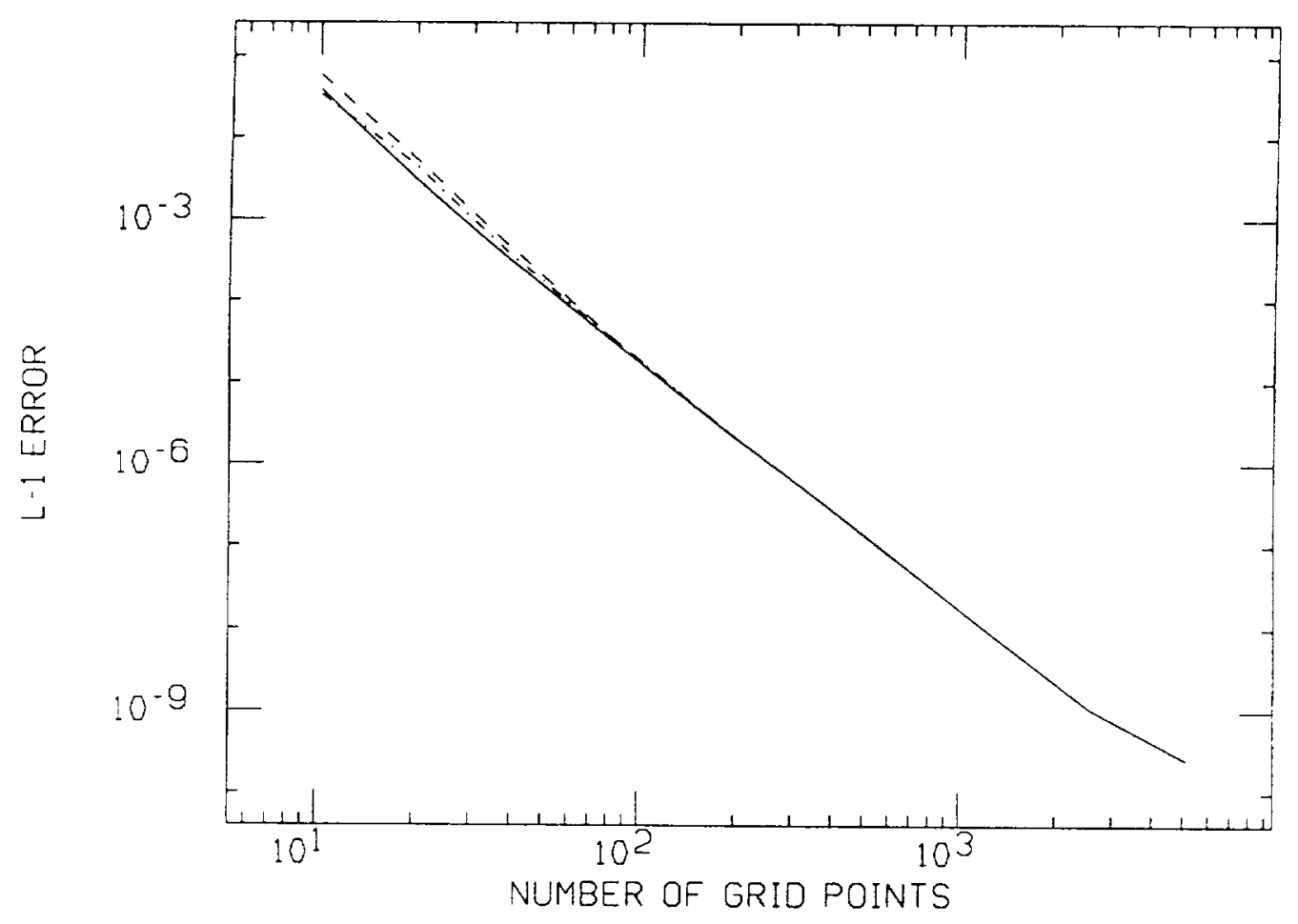

Figure 1a: $L_{1}$ error as a function of the number of grid points. $u^{0}(x)=\sin (\pi x), t=4$, $\frac{\Delta t}{\Delta x}=0.6$. Solid line: linear centered; Dashed line: ENO; Dot-Dashed line: modified ENO. Number of grid points: $10,20,40,80,160,320,640,1280,2560,5120$. Log-log scale: slope represents order of accuracy 


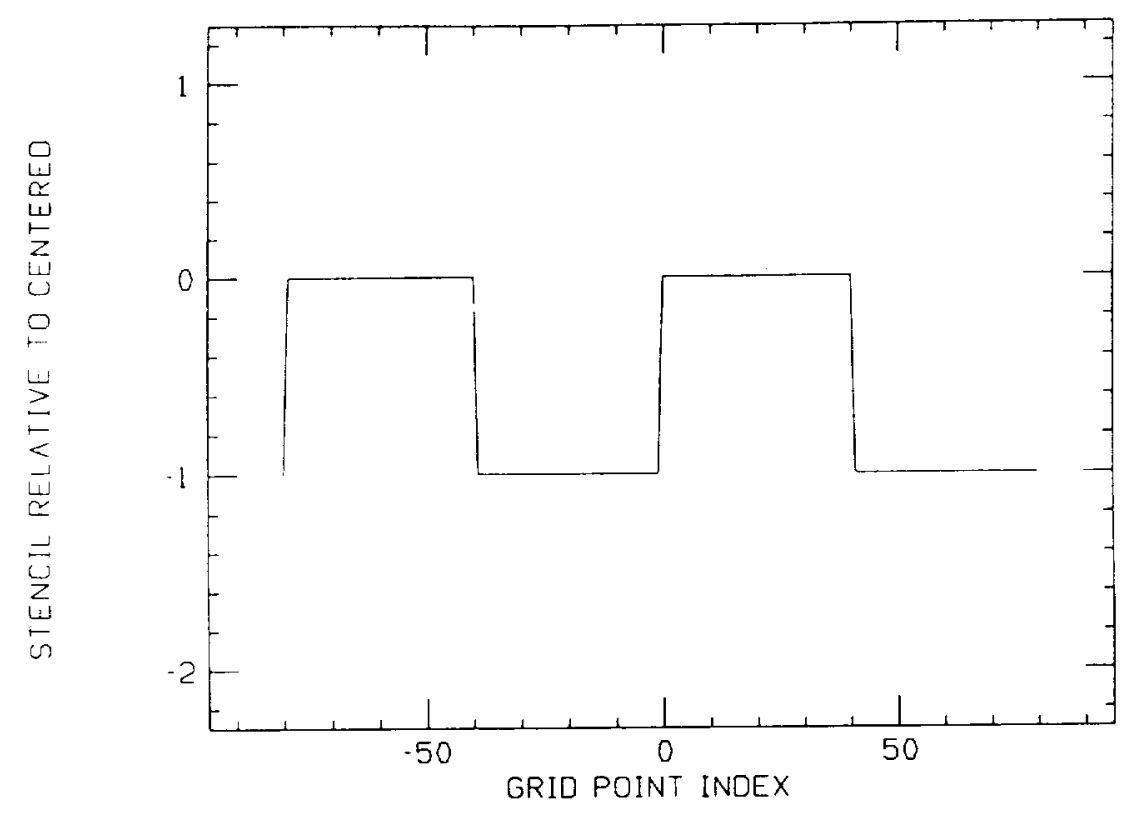

Figure 1b: The stencil choice of ENO as a function of the grid index. $u^{0}(x)=\sin (\pi x)$, $t=0$. The possible stencils for computing the flux $\hat{f}_{j+\frac{1}{2}}$ are:-2 $=\left(x_{j-3}, x_{j-2}, x_{j-1}, x_{j}\right)$; $-1=\left(x_{j-2}, x_{j-1}, x_{j}, x_{j+1}\right) ; 0=\left(x_{j-1}, x_{j}, x_{j+1}, x_{j+2}\right) ; 1=\left(x_{j}, x_{j+1}, x_{j+2}, x_{j+3}\right) ; 0$ (centered) and -1 (upwinding) are linearly stable stencils, 1 and -2 are linearly unstable stencils

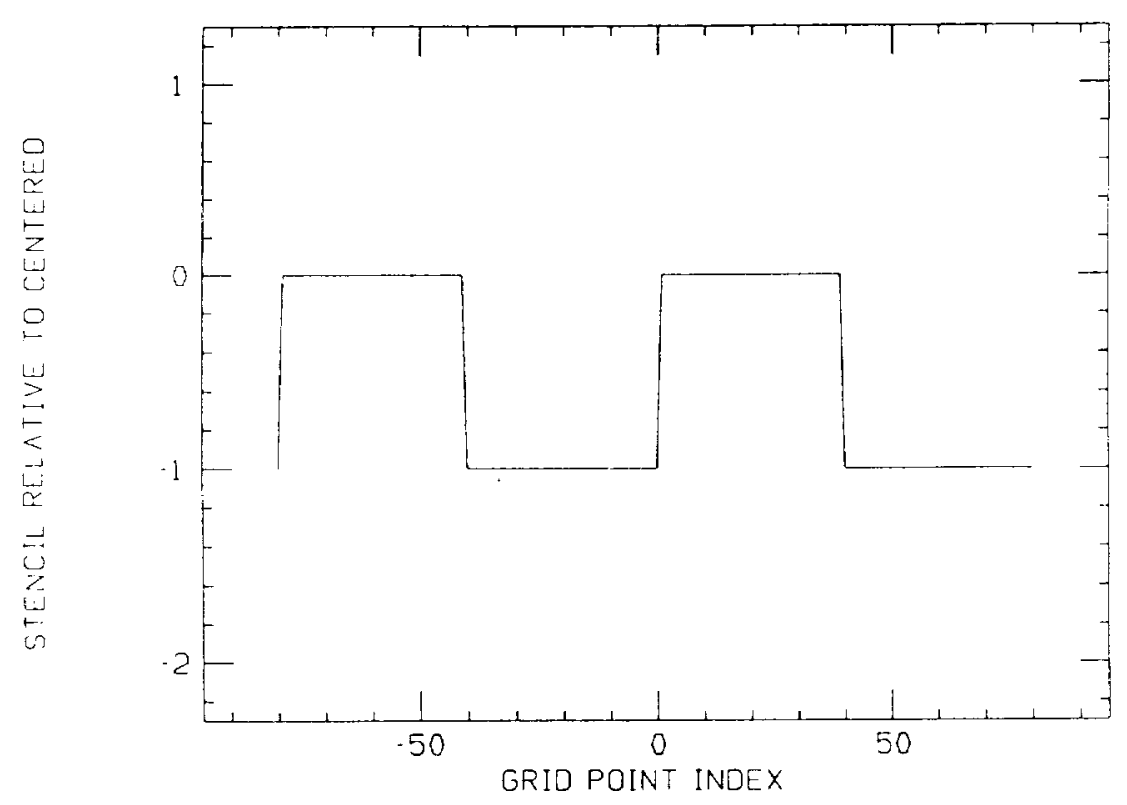

Figure 1c: same as $1 \mathrm{~b}$ except that $t=4$ 


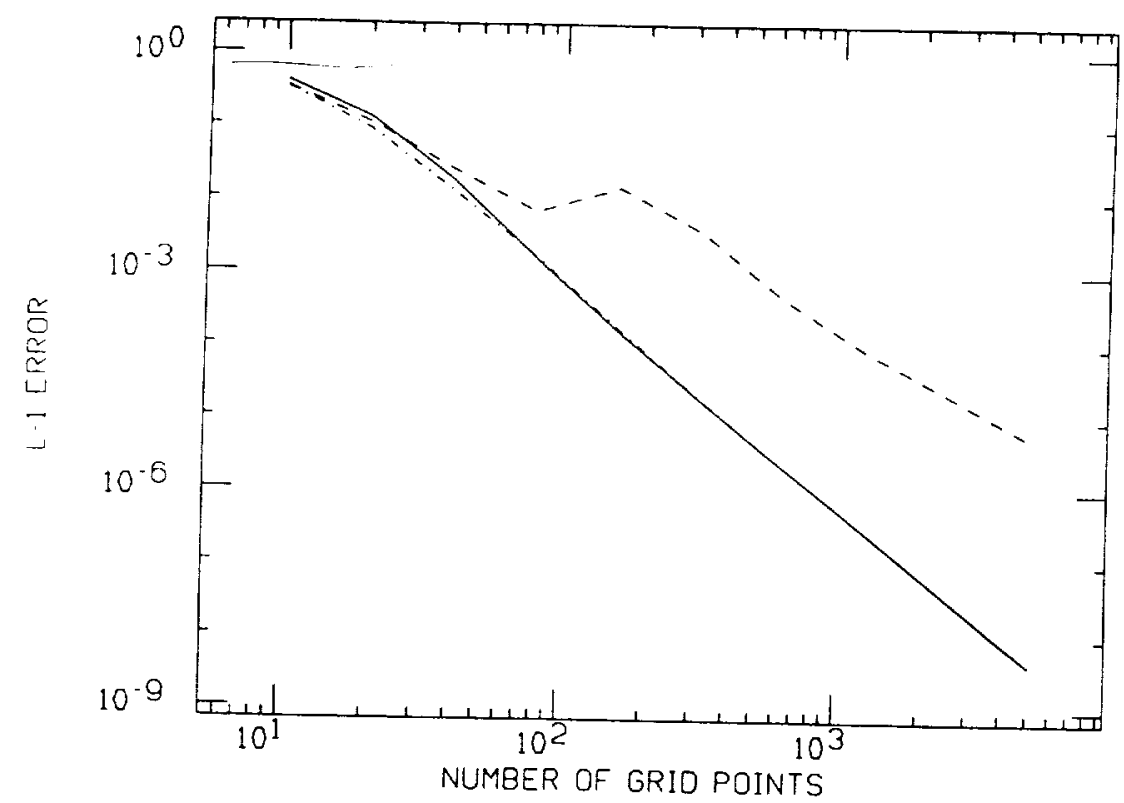

Figure 2a: same as 1 a except that $u^{0}(x)=\sin ^{4}(\pi x)$

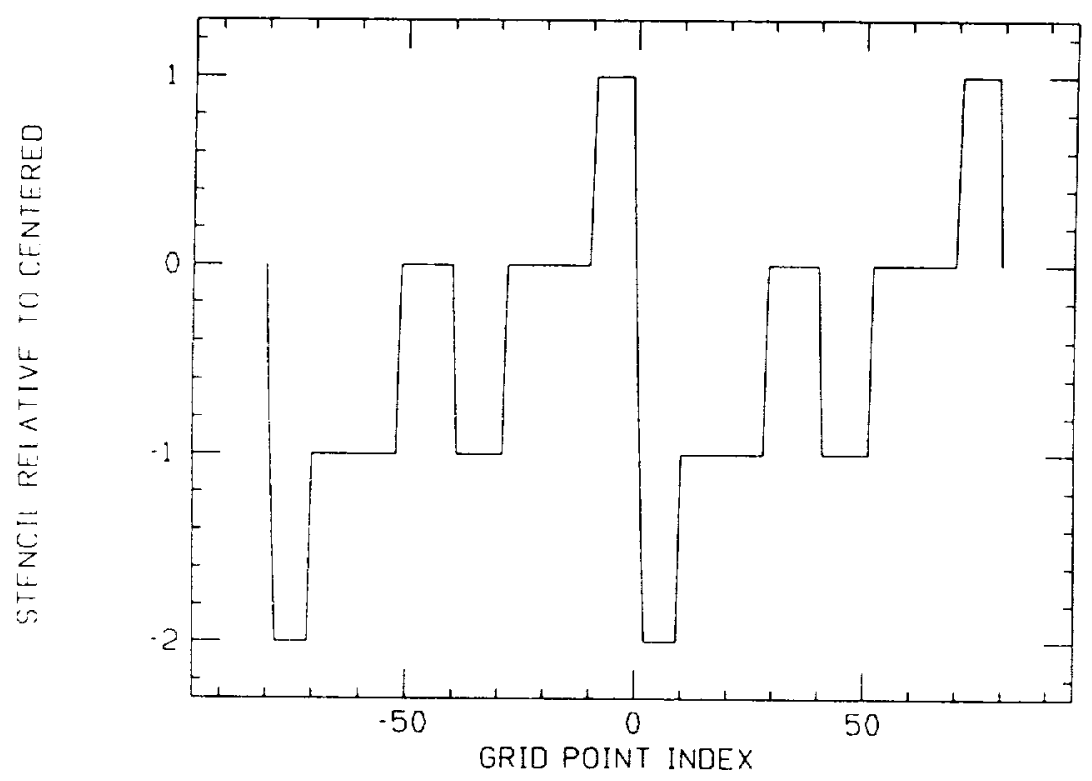

Figure 2b: same as $1 \mathrm{~b}$ except that $u^{0}(x)=\sin ^{4}(\pi x)$ 


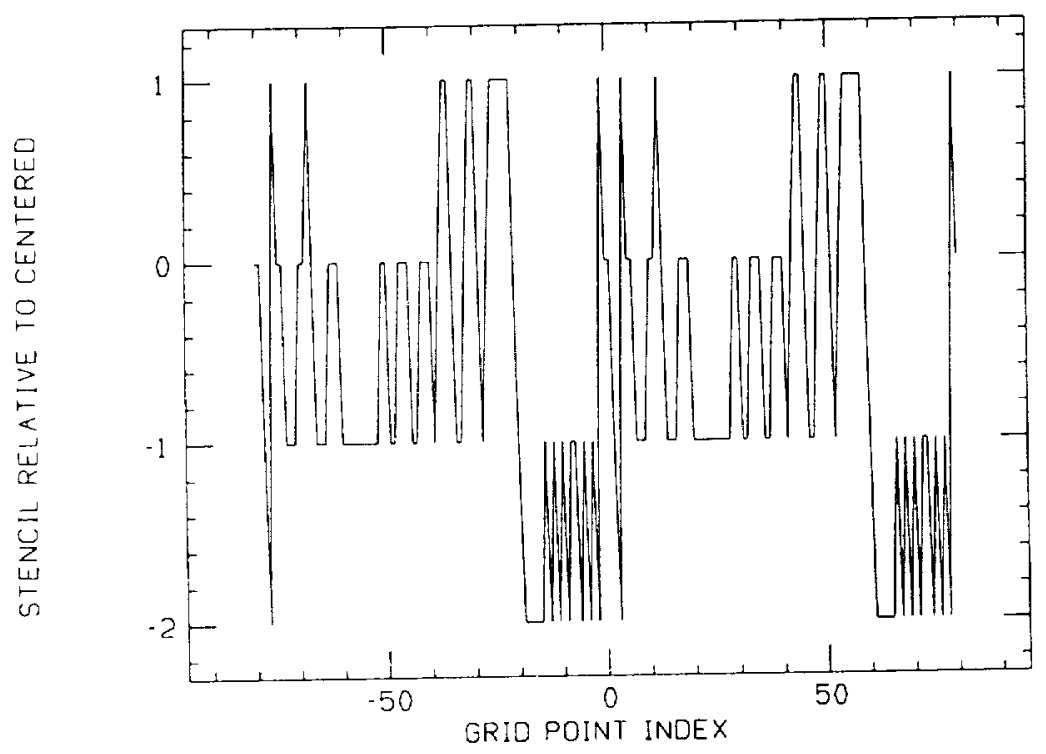

Figure 2c: same as $2 \mathrm{~b}$ except that $t=100 \Delta t$

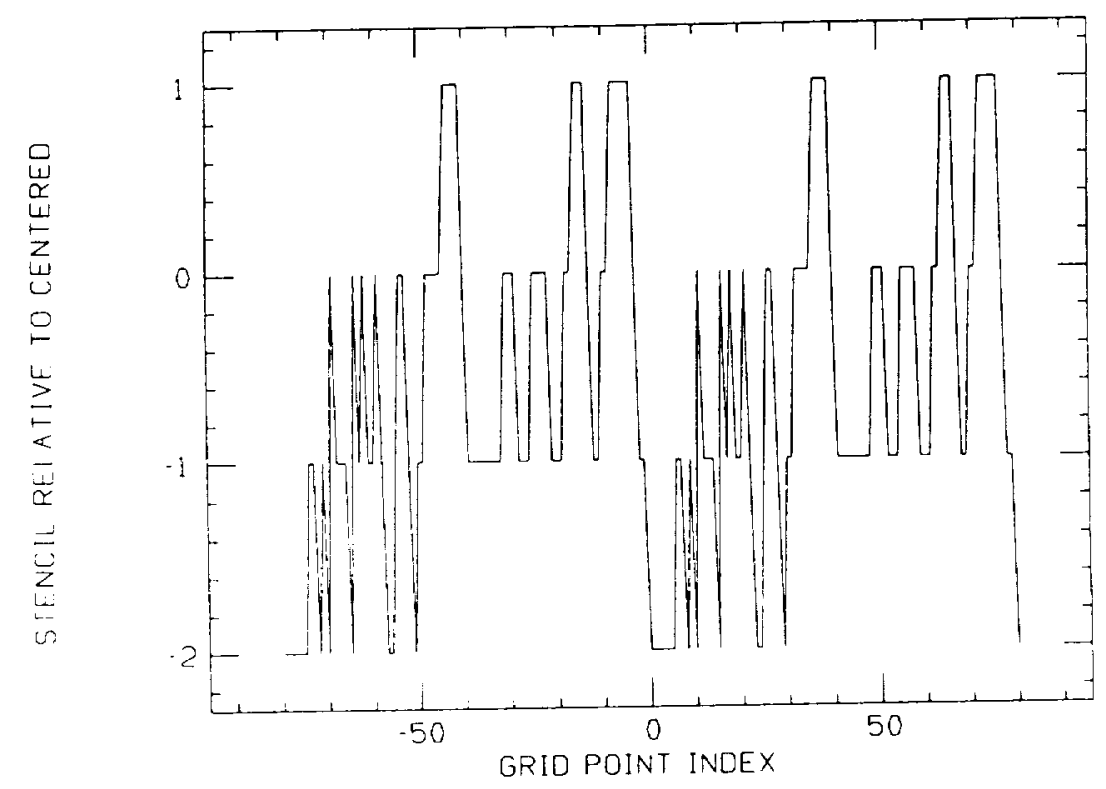

Figure 2d: same as $2 \mathrm{~b}$ except that $t=4$ 


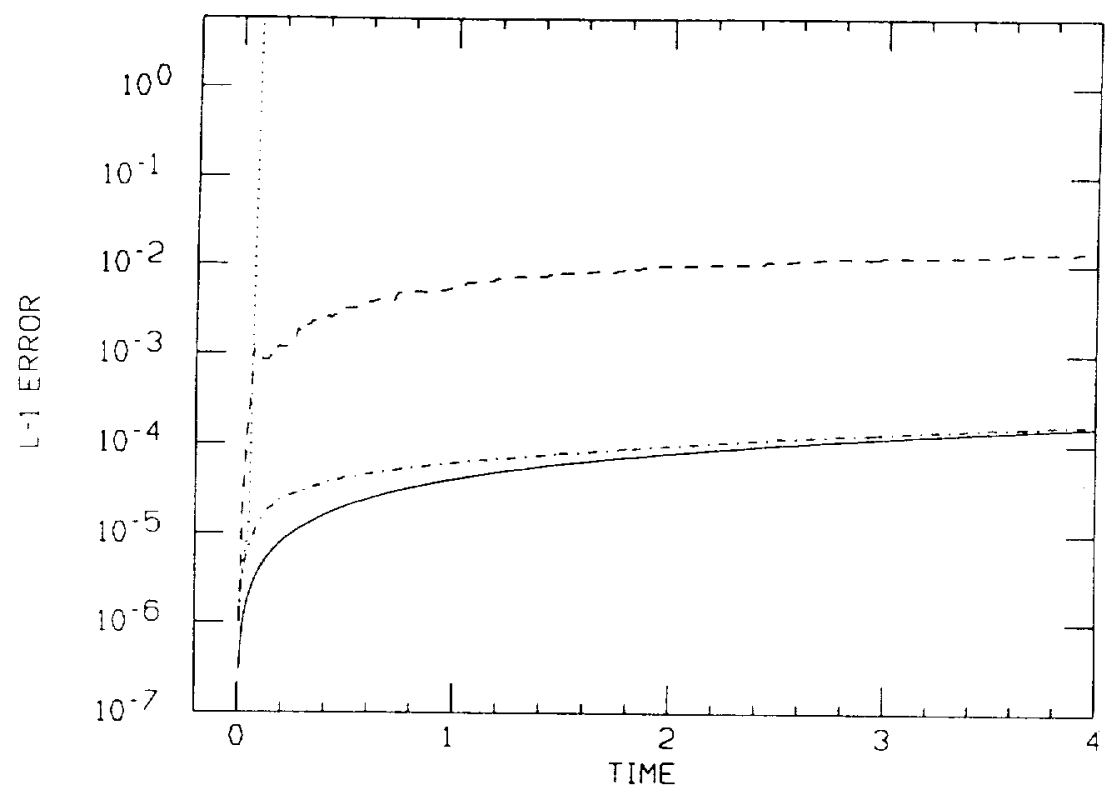

Figure 2e: $L_{1}$ error, in logrithm scale, as a function of time t, for $u^{0}(x)=\sin ^{4}(\pi x)$, with 160 grid points. Solid line: linear centered; Dashed line: ENO; Dot-Dashed line: modified ENO; Dotted line: linearly unstable scheme

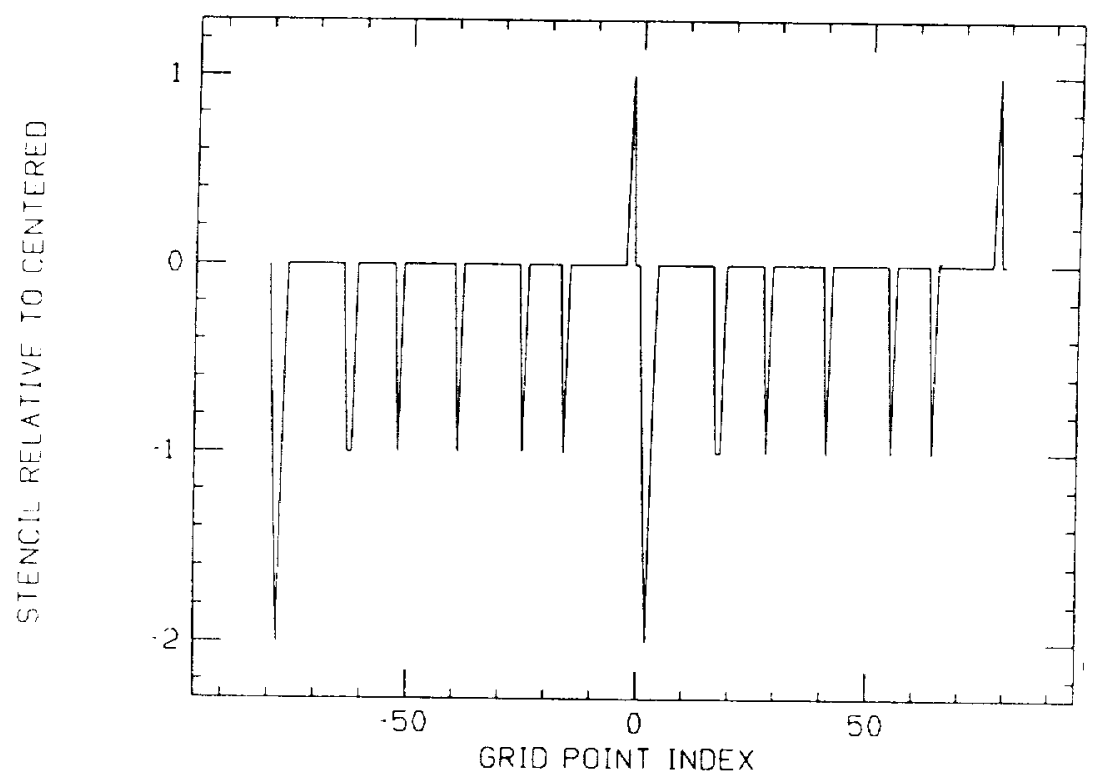

Figure 2f: same as $2 \mathrm{~b}$ except that the modified ENO is used 


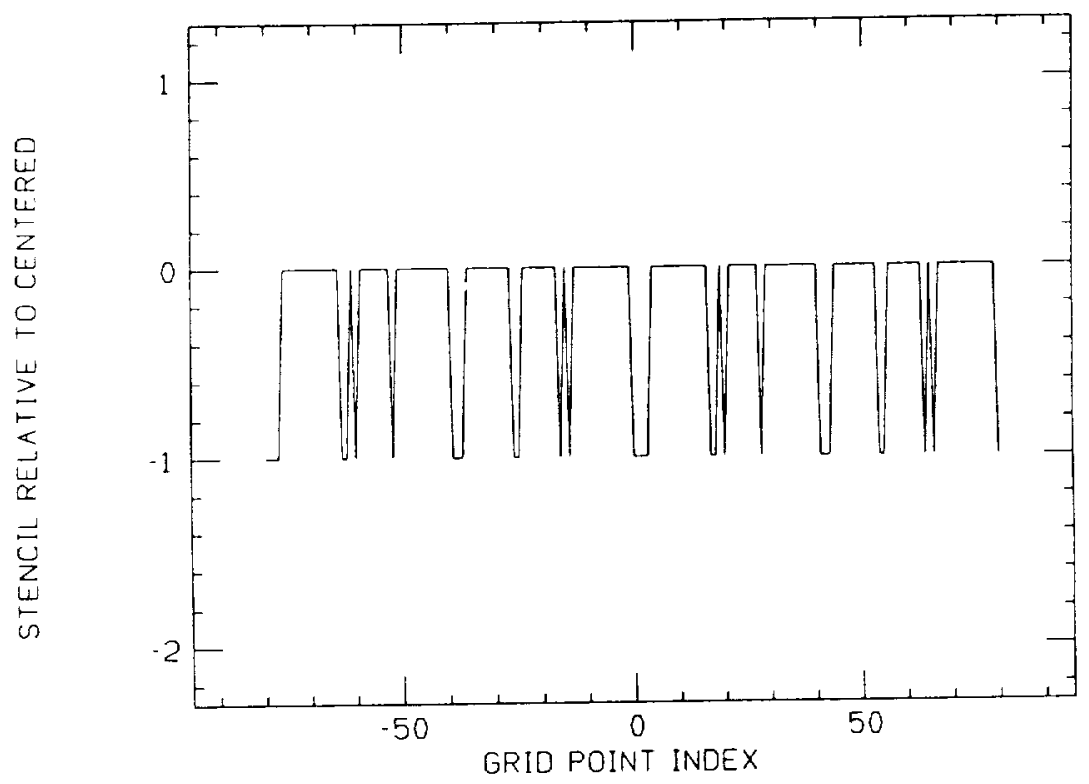

Figure 2g: same as $2 \mathrm{f}$ except that $t=4$

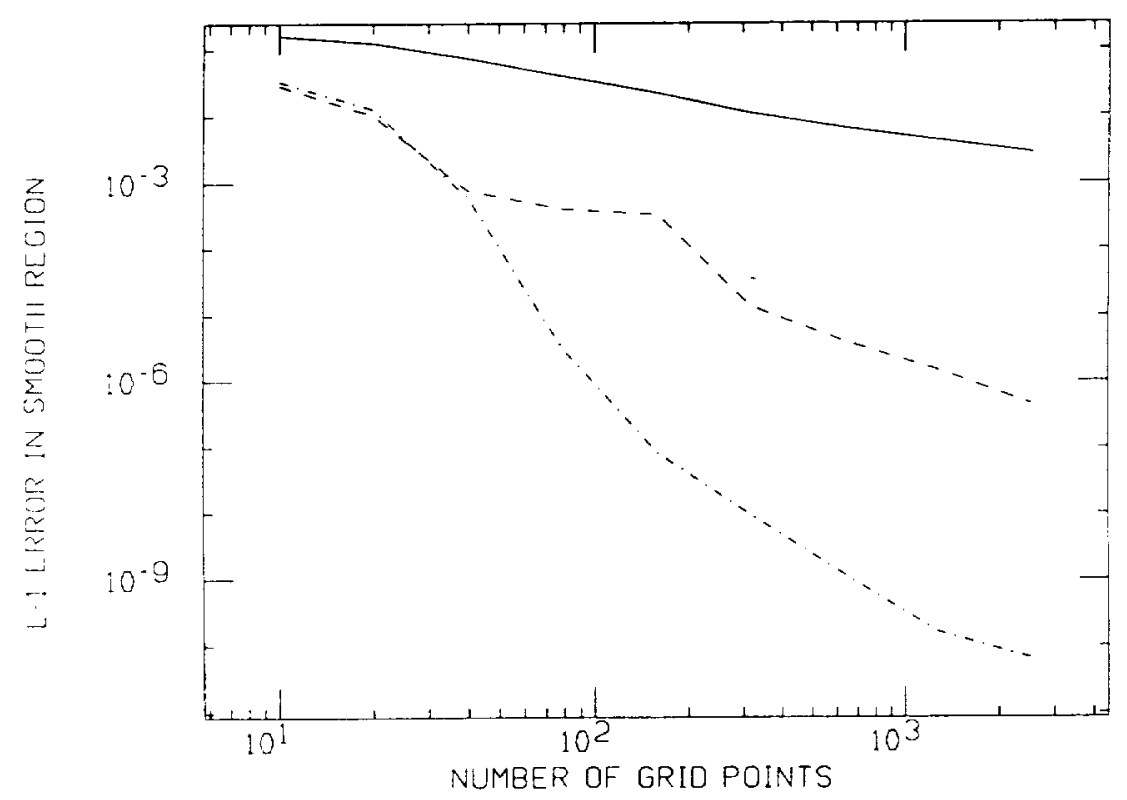

Figure 3a: same as 1a except that $u^{0}(x)=e^{-x}$ and the $L_{1}$ error is computed in the smooth region $-0.5 \leq x \leq 0.5$ 


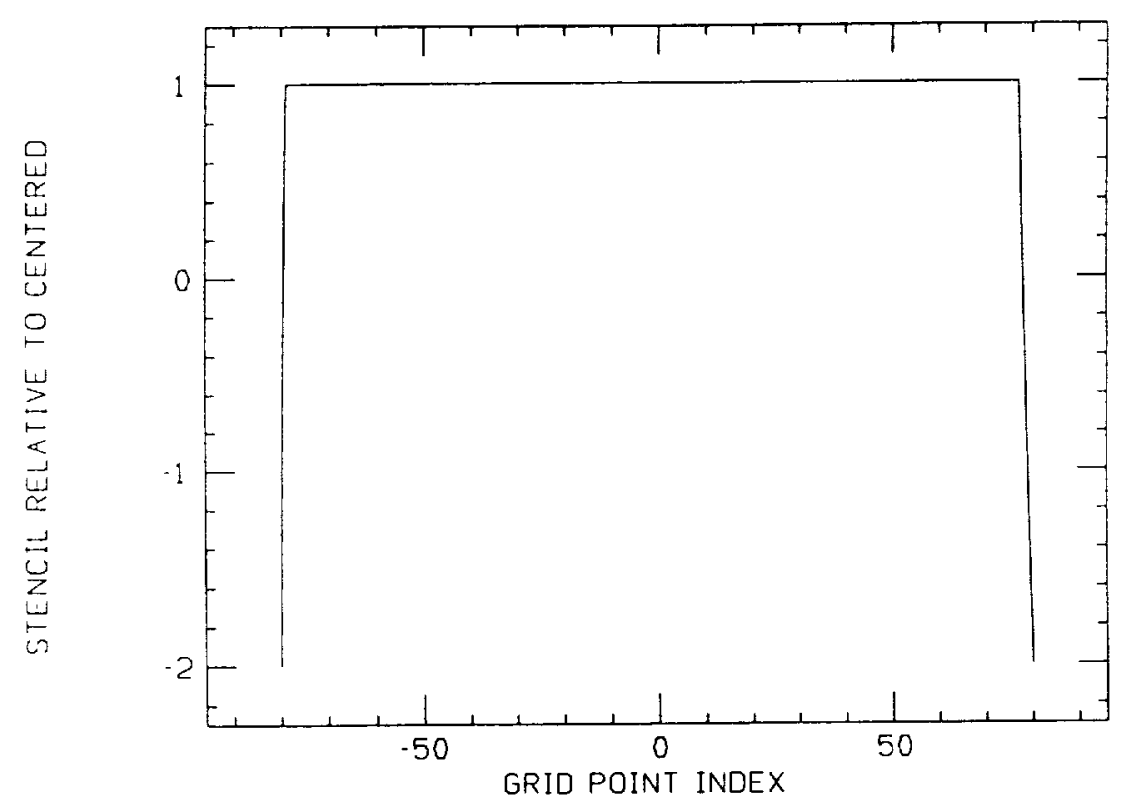

Figure $3 \mathrm{~b}:$ same as $1 \mathrm{~b}$ except that $u^{0}(x)=e^{-x}$

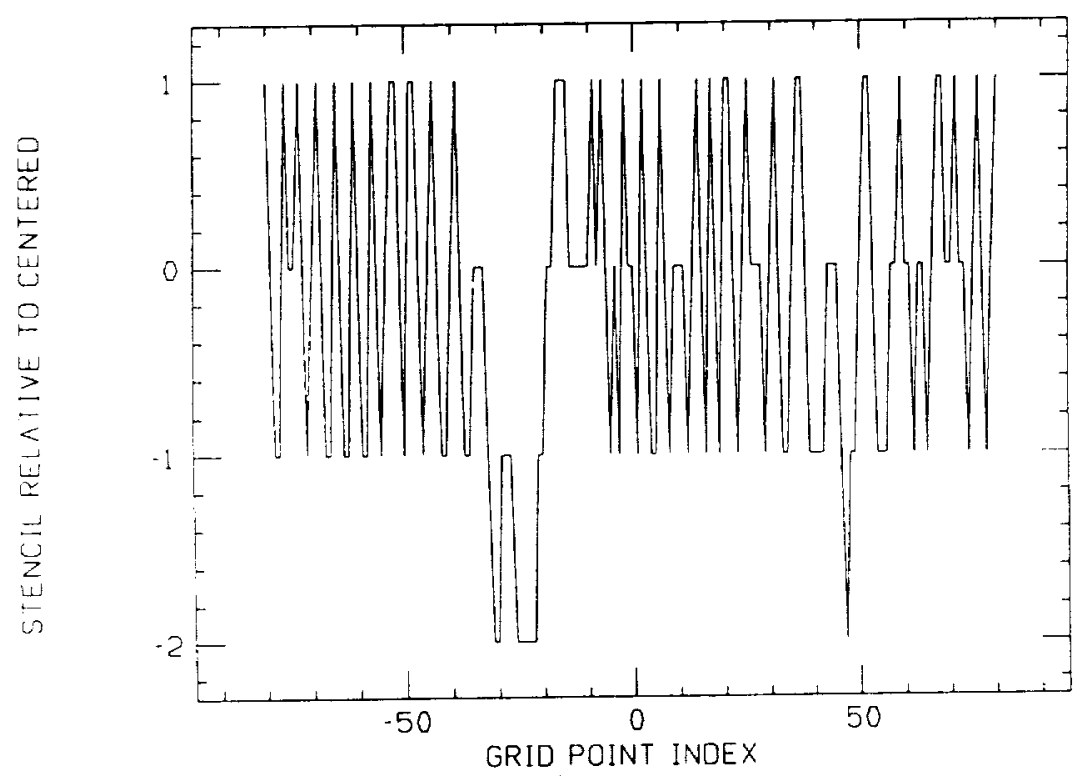

Figure 3c: same as $3 \mathrm{~b}$ except that $t=100 \Delta t$ 


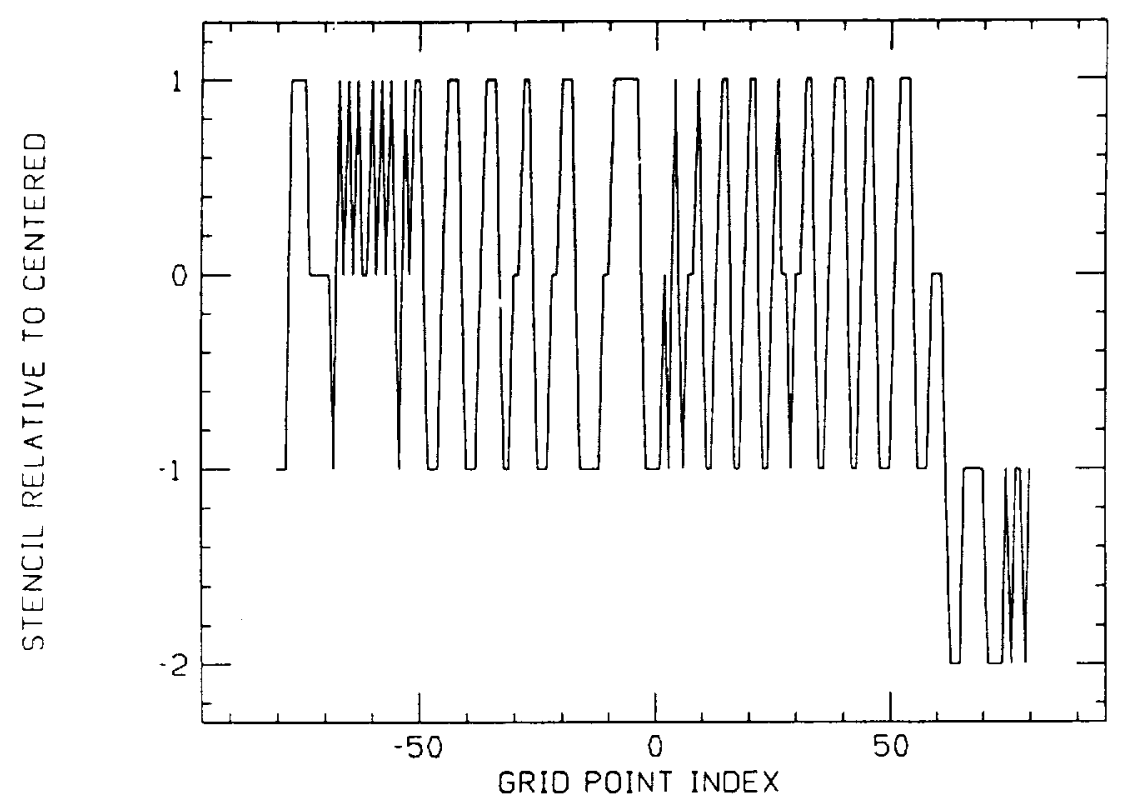

Figure 3d: same as $3 \mathrm{~b}$ except that $t=4$

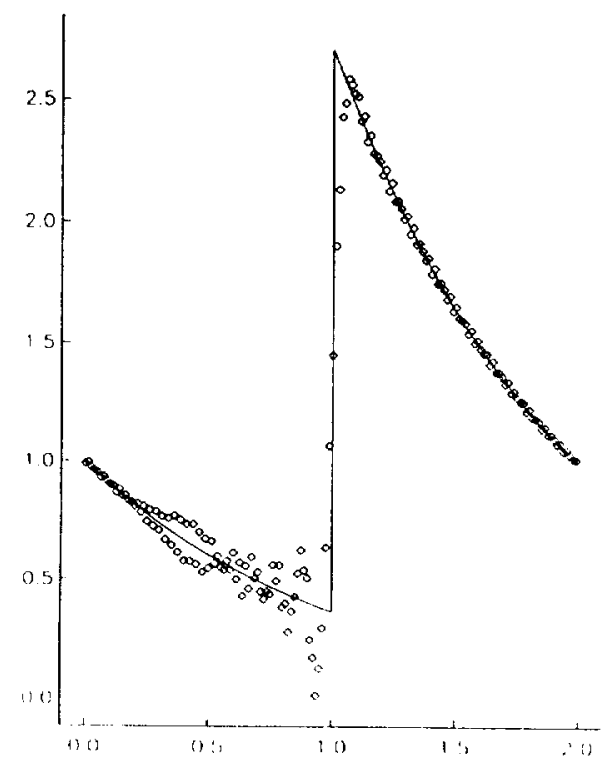

Figure 3e: $t=4, u^{0}(x)=e^{-x}$. Solid line: exact solution; Circles: solution of the linear centered scheme 


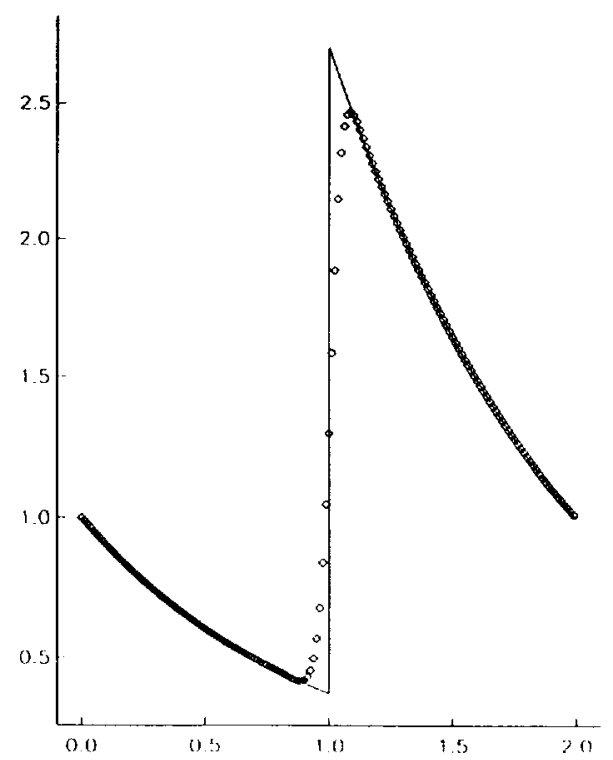

Figure 3f: same as 3e except that Circles: solution of ENO scheme

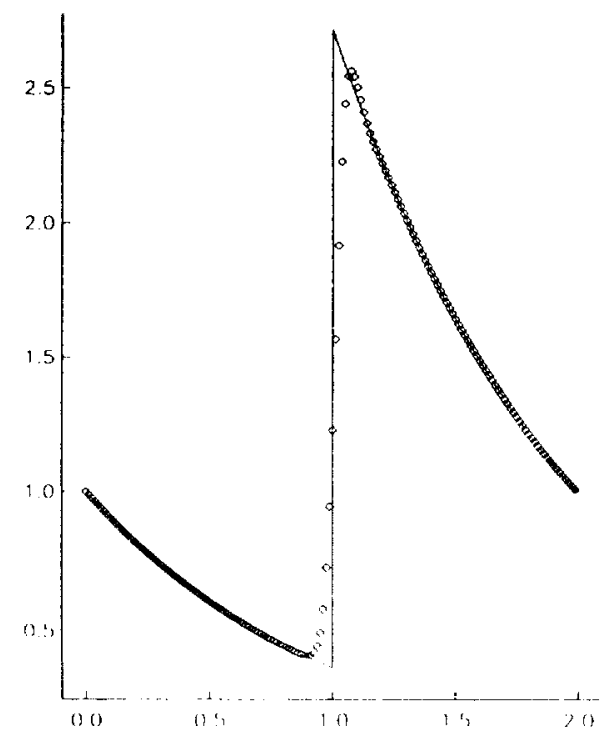

Figure 3g: same as 3e except that Circles: solution of the modified ENO scheme 

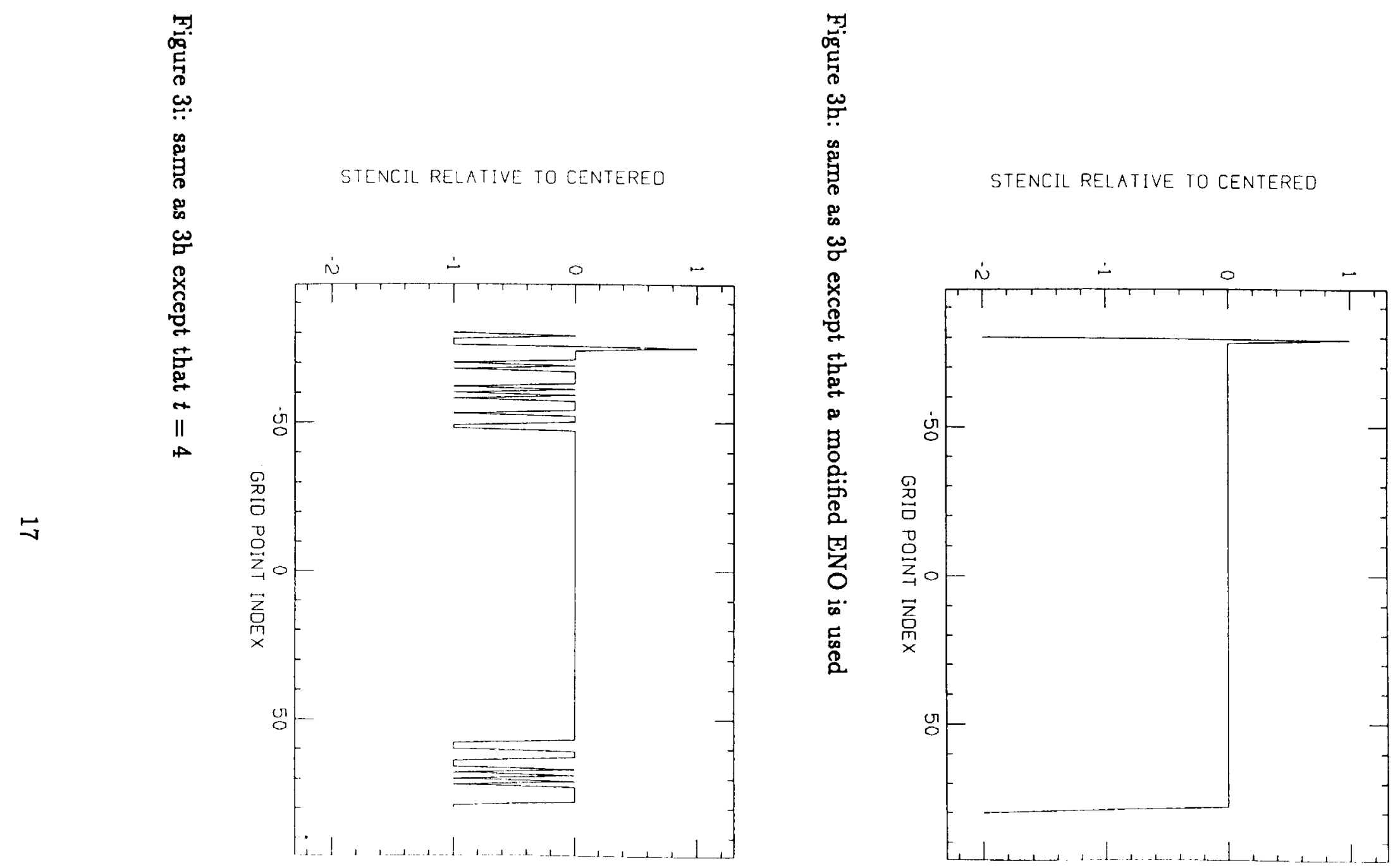


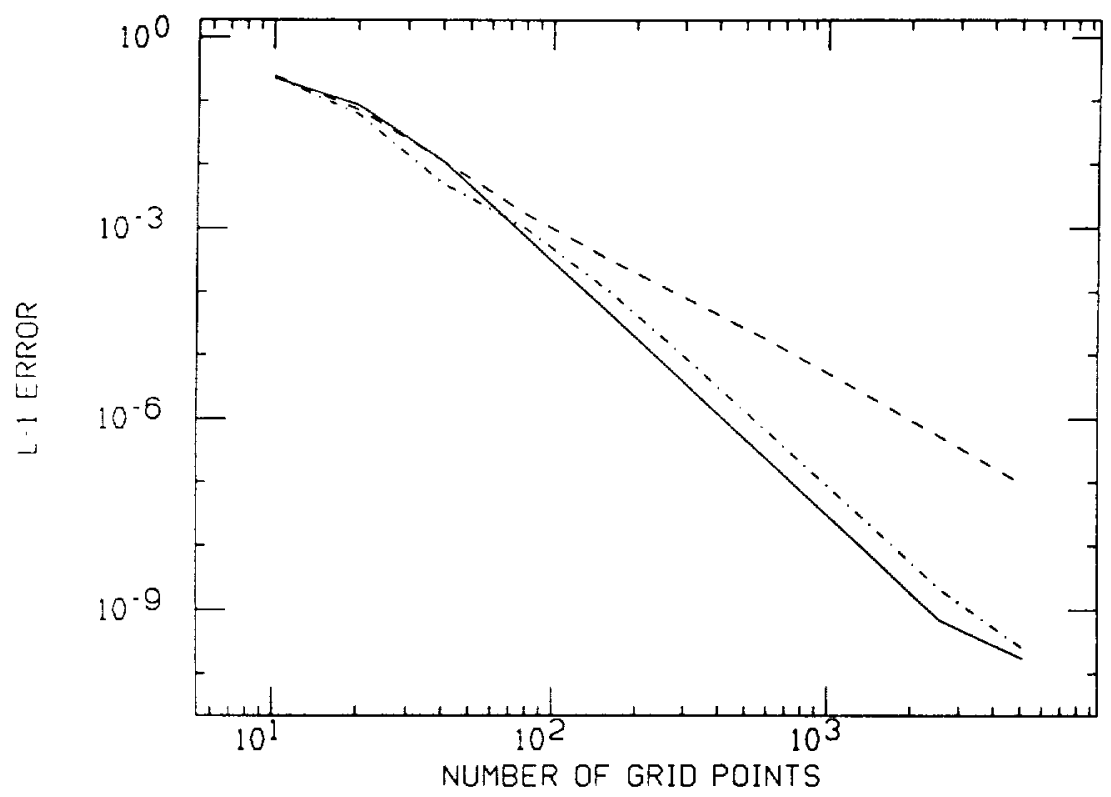

Figure 4a: same as 2a except that the exact time evolution is used

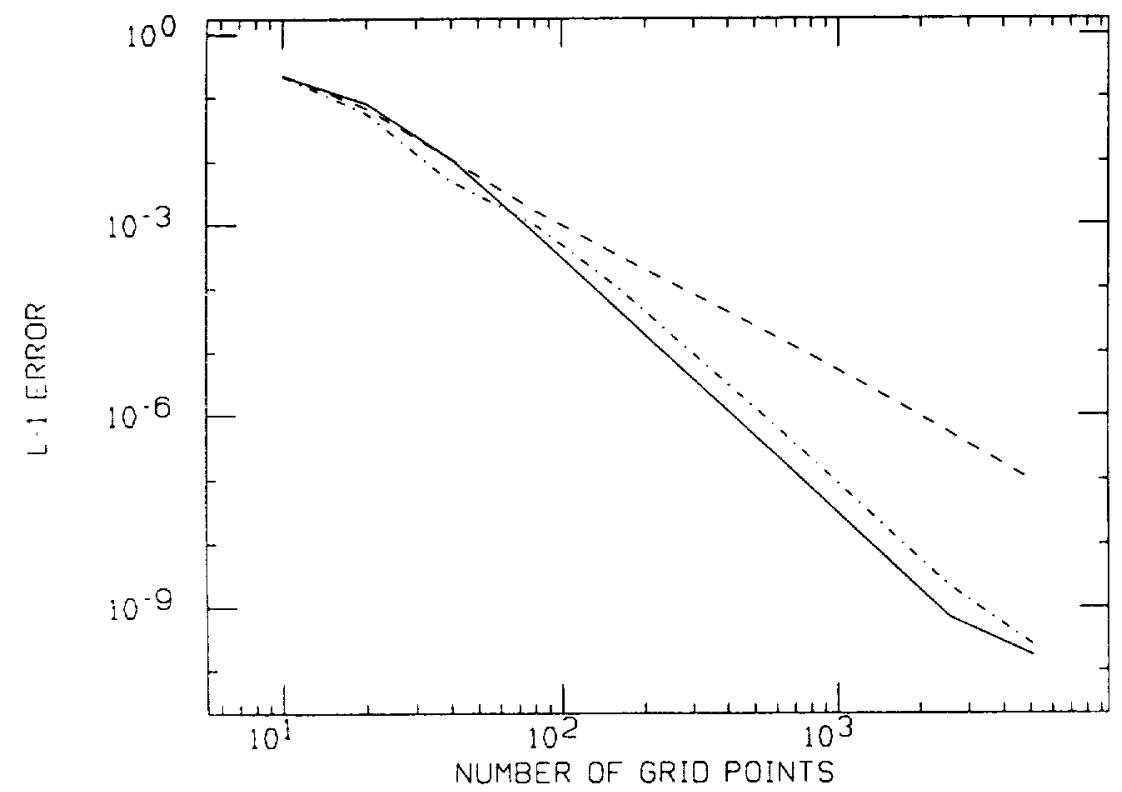

Figure 4b: same as 4a except that the cell-averaged ENO is used 


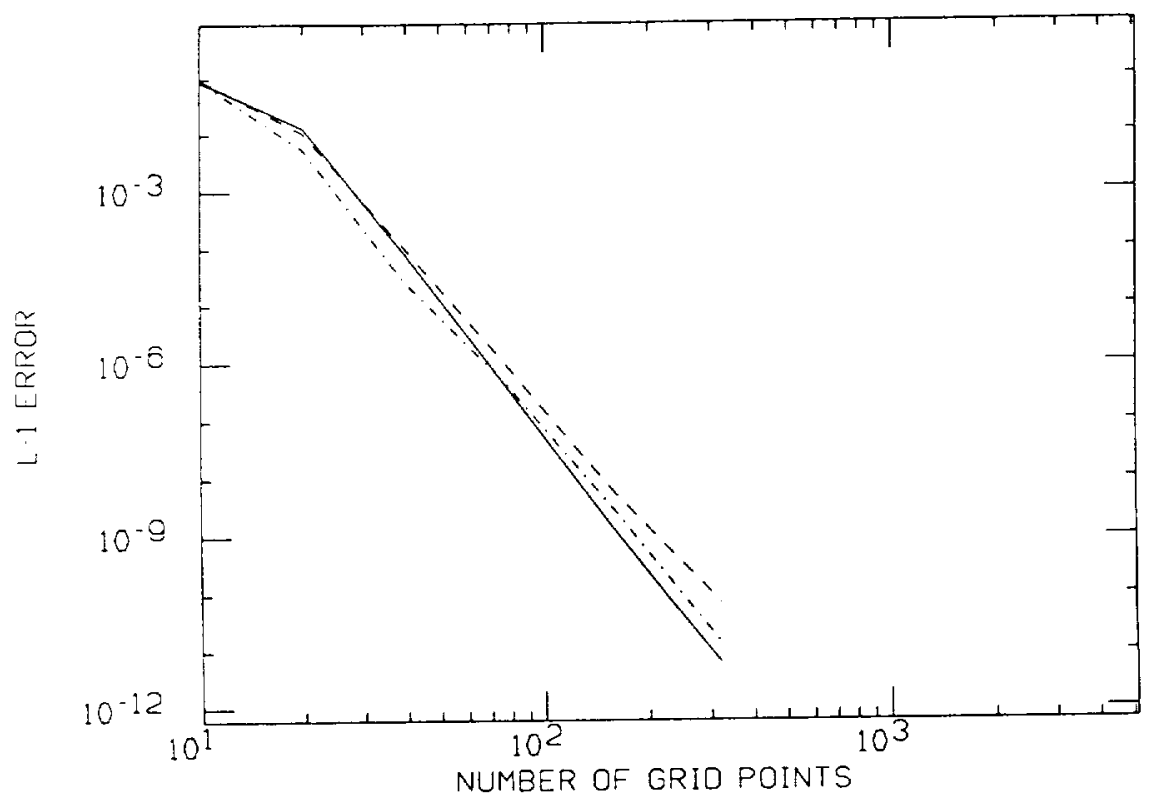

Figure 4c: same as 4a except that seventh order in space is used

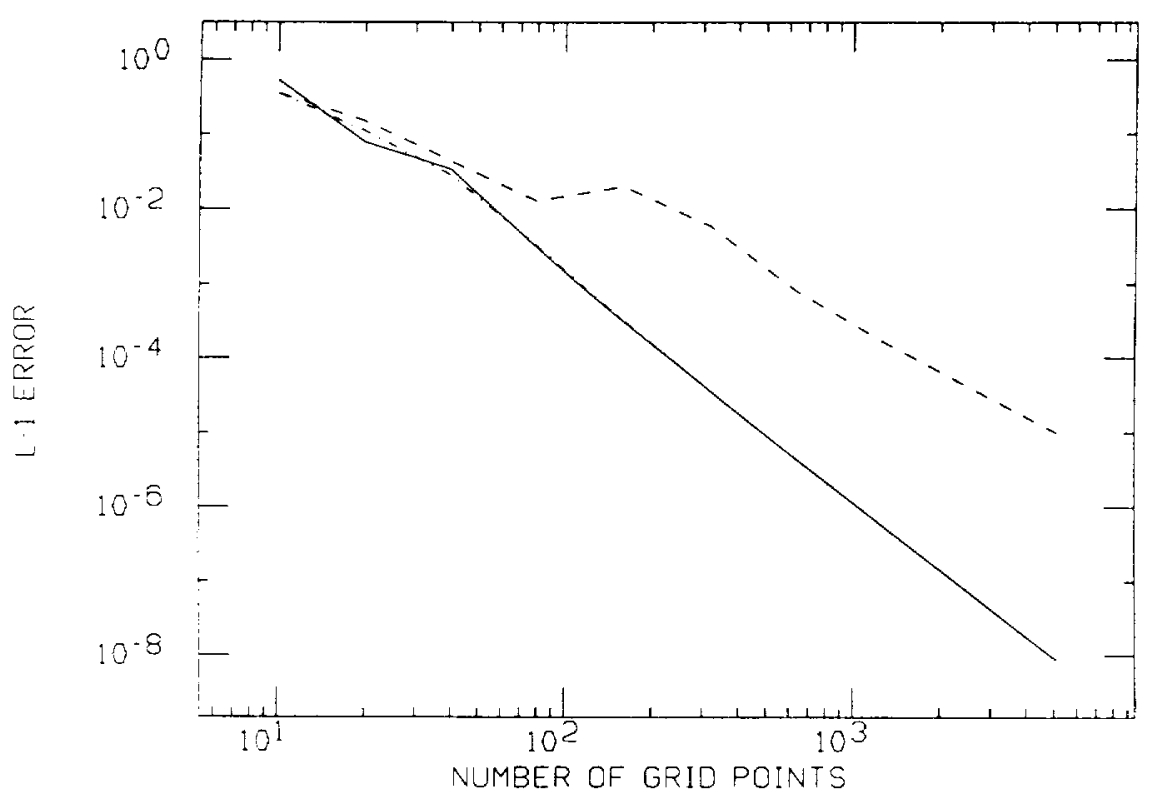

Figure 4d: same as 2a except that $t=8$ 


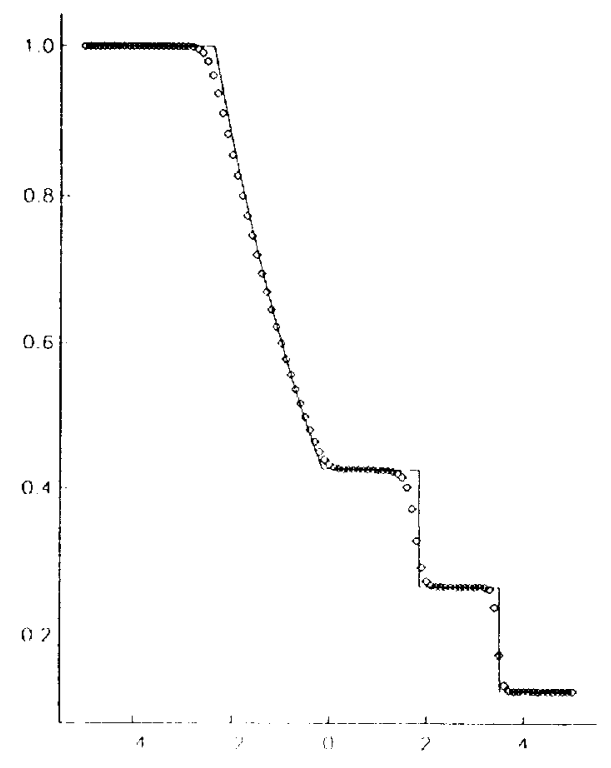

Figure 5a: shock tube problem with $\left(\rho_{L}, u_{L}, p_{L}\right)=(1,0,1),\left(\rho_{R}, u_{R}, p_{R}\right)=(0.125,0,0.1)$. Density is plotted. 100 grid points. Solid line: exact solution; Circles: ENO

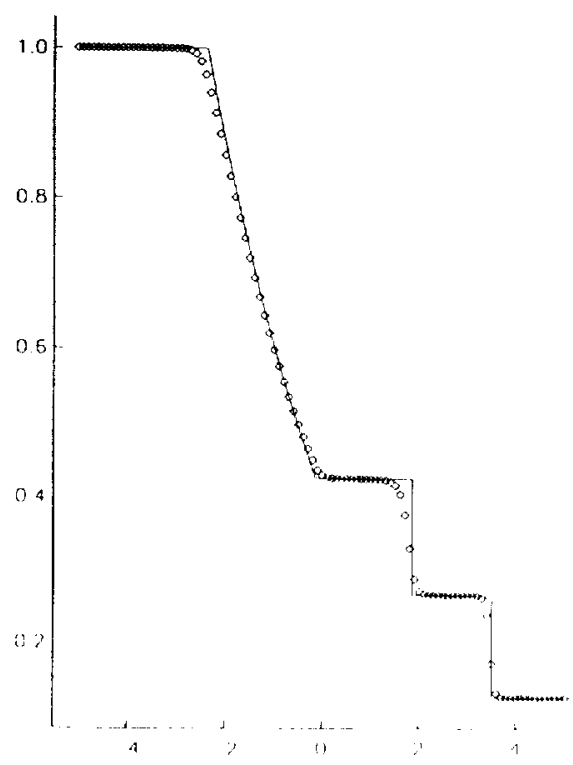

Figure 5b: same as 5 a except that Circles: modified ENO 


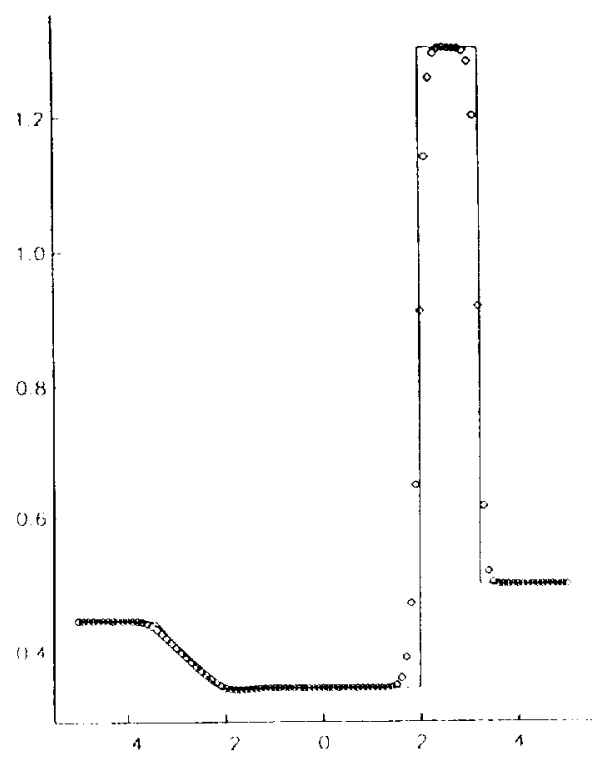

Figure 6a: same as 5a except that $\left(\rho_{L}, u_{L}, p_{L}\right)=(0.445,0.698,3.528),\left(\rho_{R}, u_{R}, p_{R}\right)=(0.5,0,0.571)$

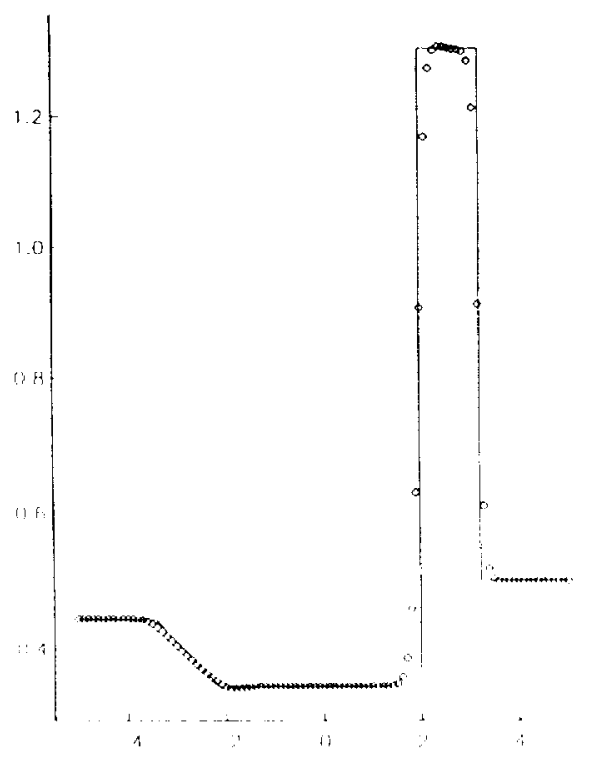

Figure 6b: same as 6a except that Circles: modified ENO 


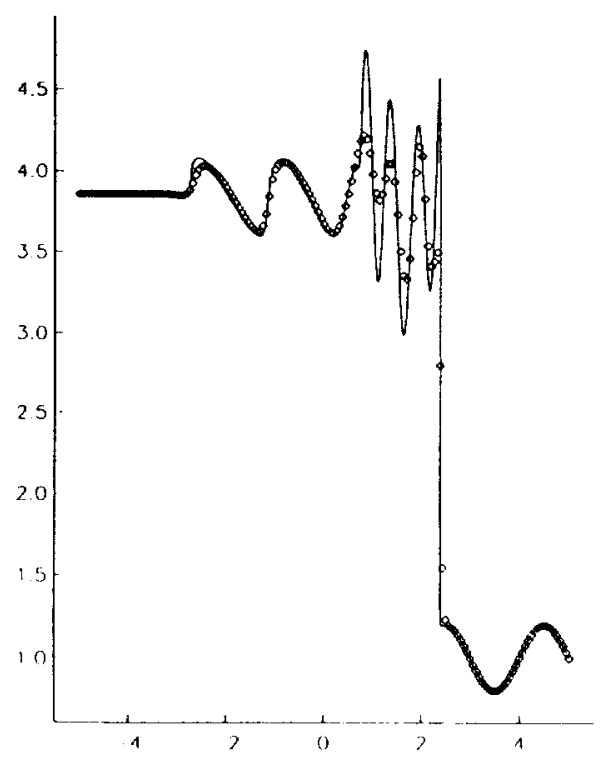

Figure 7a: shock interaction with density perturbations. $\left(\rho_{L}, u_{L}, p_{L}\right)=(3.857143,2.629369,10.333333)$, $\left(\rho_{R}, u_{R}, p_{R}\right)=(1+0.2 \sin (5 x), 0,1)$. Density is plotted. Solid line: a converged solution computed by ENO with 1200 grid points; Circles: ENO with 160 grid points

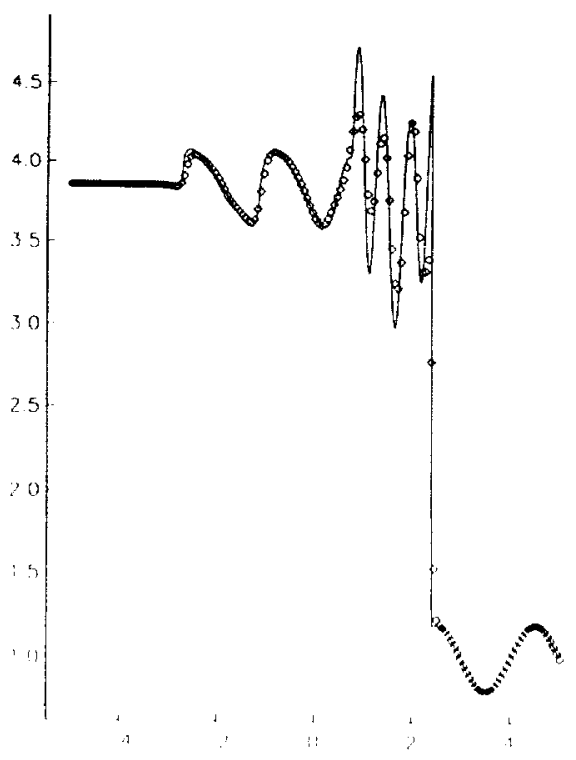

Figure 7b: same as 7a except that Circles: modified ENO with 160 grid points 


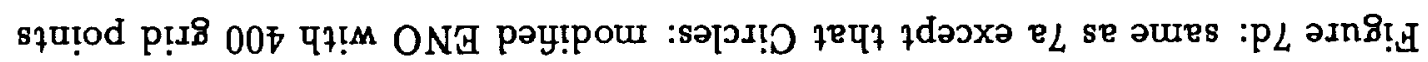

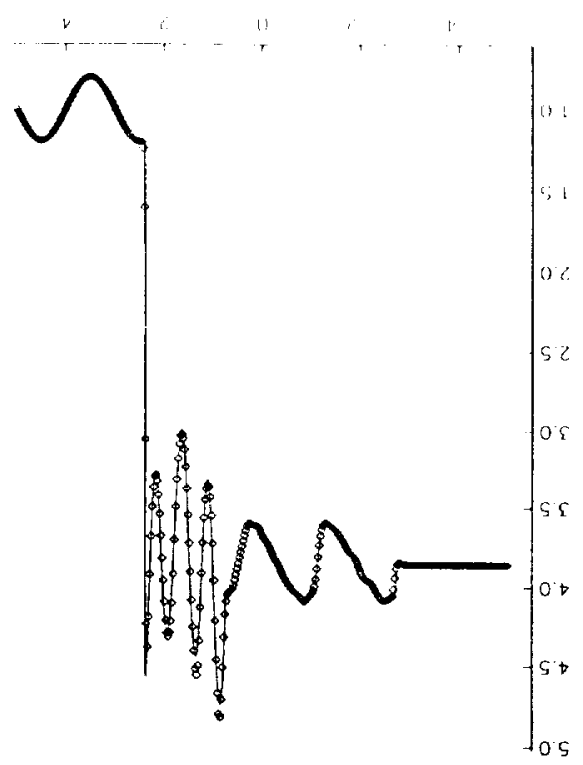

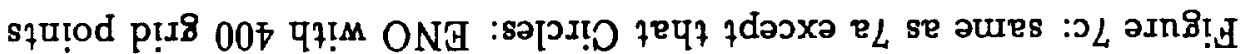

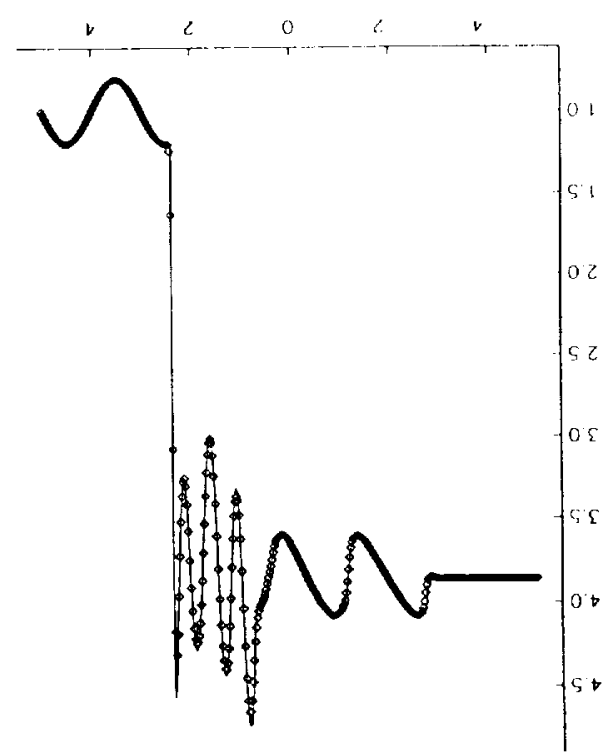





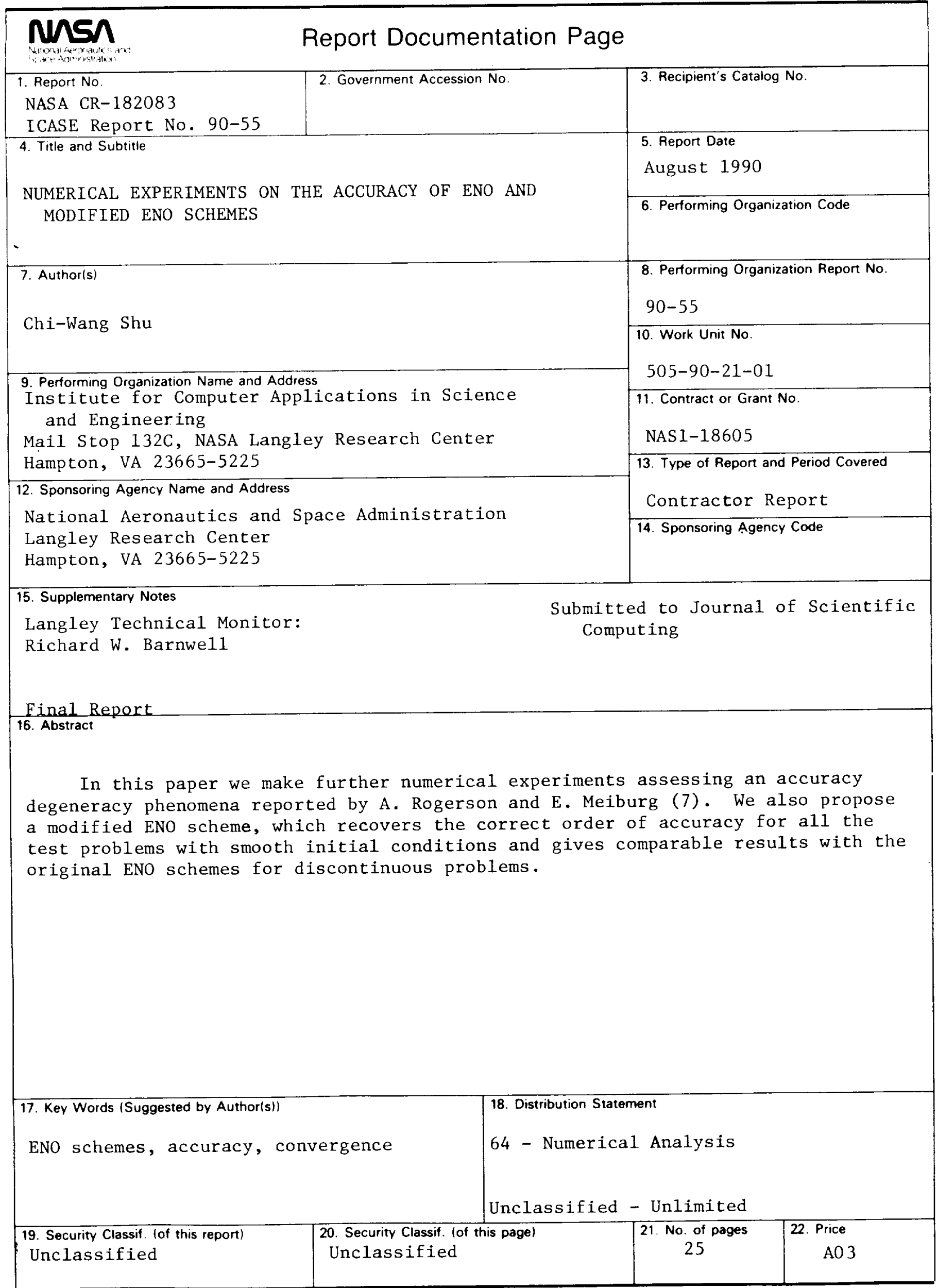


